\title{
Neglect and aphasia in the acute phase as predictors of functional outcome 7 years after ischemic stroke
}

\author{
J. Gerafi, ${ }^{a, b, d, e}$ (iD, H. Samuelsson ${ }^{a, b}$, J. I. Viken ${ }^{a, b}$, C. Blomgren ${ }^{b}$, L. Claesson ${ }^{b}$, S. Kallio ${ }^{d}$, C. Jern ${ }^{c}$, \\ C. Blomstrand ${ }^{b}$ and K. Jood ${ }^{b, f}$
}

\begin{abstract}
${ }^{a}$ Department of Psychology, Faculty of Social Sciences, University of Gothenburg, Gothenburg; ${ }^{\mathrm{b}}$ Institute of Neuroscience and Physiology, The Sahlgrenska Academy at University of Gothenburg, Gothenburg; ${ }^{\mathrm{c}}$ Institute of Biomedicine, The Sahlgrenska Academy at University of Gothenburg, Gothenburg; ${ }^{\mathrm{d} D e p a r t m e n t ~ o f ~ C o g n i t i v e ~ N e u r o s c i e n c e ~ a n d ~ P h i l o s o p h y, ~ I n s t i t u t e ~ o f ~ B i o s c i e n c e, ~ U n i v e r s i t y ~ o f ~ S k o ̈ v d e, ~}$ Skövde; ${ }^{\mathrm{e}}$ The Skaraborg Institute for Research and Development, Skövde; and ${ }^{\mathrm{f}}$ Department of Neurology, The Sahlgrenska University Hospital, Gothenburg, Sweden
\end{abstract}

\section{Keywords:}

ischemic stroke, language impairment, long-term functional outcome, visuospatial inattention

Received 6 December 2016 Accepted 8 August 2017

European Journal of Neurology 2017, 0: 1-9 doi:10.1111/ene.13406
Background and purpose: Visuospatial inattention (VSI) and language impairment (LI) are often present early after stroke and associations with an unfavorable short-term functional outcome have been reported. The purpose of this study was to investigate whether a screening of VSI and LI as indicators of cortical symptoms early after stroke could predict long-term functional outcomes.

Methods: A consecutive cohort of 375 patients with ischemic stroke was assessed for the occurrence of VSI at a median of 7 days after admission (interquartile range, 1-5 days) using the Star Cancellation Test and for LI (within the first 7 days) with the language item in the Scandinavian Stroke Scale. Seven years later, functional outcomes were assessed by the modified Rankin scale and Frenchay Activities Index in 235 survivors without recurrent stroke. Relationships between baseline predictors and functional outcome at 7 years were analyzed with bivariate correlations and multiple categorical regressions with optimal scaling.

Results: The regression model significantly explained variance in the modified Rankin scale $\left(R^{2}=0.435, P<0.001\right)$ and identified VSI $(P=0.001)$ and neurological deficits $(P<0.001$; Scandinavian Stroke Scale score without the language item) as the significant independent predictors. The model for Frenchay Activities Index was also significant $\left(R^{2}=0.269, \quad P<0.001\right)$ with VSI $(P=0.035)$ and neurological deficits $(P<0.001)$ as significant independent predictors.

Conclusions: Visuospatial inattention at acute stroke has an independent impact on long-term functional outcomes. Early recognition may enable targeted rehabilitative interventions.

\section{Introduction}

Neglect and aphasia are common clinical symptoms at the early stage after stroke [1-5] and numerous studies have identified significant relationships between these symptoms and unfavorable functional outcomes when patients were followed over the first 6-12 months

Correspondence: J. Gerafi, Department of Psychology, University of Gothenburg, Box 500, 40530 Gothenburg, Sweden

(tel.: + 467037 67553; fax: + 463178 64628;

e-mail: joel.gerafi@psy.gu.se). post-stroke [6-14]. However, little is known about the long-term effects of these early symptoms. To our knowledge, there are no studies of both neglect and aphasia as potential predictors of long-term functional outcome after the first year post-stroke.

In this study, our aim was to investigate whether a basic screening of visuospatial inattention (VSI) and language impairment (LI) at the early stage poststroke could provide prognostic information about long-term functional outcome. The study focused on a cohort of relatively young stroke survivors. Increased 
knowledge of long-term outcomes in this group of individuals is of specific importance as they have long life expectancy and may live many years with strokerelated consequences during a period normally devoted to an active life. Reliable information about the long-term prognosis is of importance, not only for the affected individuals and families, but also for the design of long-term intervention and support programs. We hypothesized that basic information about impairments in visuospatial attention and language, at the early stage after stroke, could predict functional outcome, i.e. disability and levels of participation in activities of daily living, 7 years post-stroke.

\section{Methods}

\section{Participants}

The participants were from the Sahlgrenska Academy Study of Ischemic Stroke (SAHLSIS). SAHLSIS is a longitudinal case-control study of ischemic stroke in the young and middle-aged adults, for which the details have been described elsewhere [15]. In brief, patients who presented with first-ever or a recurrent acute ischemic stroke between the ages of 18 and 69 years were consecutively recruited at stroke units in western Sweden. The present study comprises the SAHLSIS participants who were recruited at the stroke unit at the Sahlgrenska University Hospital between August 1998 and December $2003(n=375)$. They were investigated at day 1-7 and 7 years after stroke. Recurrent strokes were identified by medical history at the 7-year visit and by using the National Inpatient Register as described previously [16]. In this study, patients who had suffered from a recurrent stroke during the follow-up period were excluded.

The Regional Ethics Review Board at the University of Gothenburg approved this study. Written informed consent was obtained from all participants. For those who were unable to communicate, consent was obtained from their next of kin.

\section{Baseline predictor variables}

\section{Visuospatial inattention}

The investigation of VSI included both asymmetric neglect and non-asymmetric inattention, and was assessed with the Star Cancellation Test (SCT), which is a conventional paper-and-pencil subtest of the normalized and standardized Behavioral Inattention Test with a total score of 54 . This battery has shown good reliability [17]. Patients were classified into three ordinal levels according to their performance in the test: (i) lateralized visual inattention, i.e. omission of at least three more targets at one half of the SCT sheet; (ii) non-lateralized visual inattention, i.e. total score $\leq 52$ and not fulfilling criteria for lateralized visual inattention; and (iii) no visual inattention, i.e. a total score $>52$. The cut-off levels in the SCT were based on the performance of 25 controls (median, 57 years; range, 29-70 years) from a previous study [18].

\section{Language impairment}

The language item of the Scandinavian Stroke Scale (SSS) [19] was used to assess the degree of LI after stroke in three ordinal levels of severity: (i) severe LI, i.e. the patient only produces yes/no or less, or the patient produces more than yes/no, but no longer sentences; (ii) mild LI, i.e. limited vocabulary or incoherent speech; and (iii) no LI, i.e. no signs of aphasia. For all participants who, according to SSS, had LI combined with either decreased level of consciousness or facial palsy, the LIs were validated against the medical records. For all included participants, this procedure resulted in reclassification of LI in eight participants as no LI.

Patients who could not be tested with the SCT or the SSS language item were retrospectively classified with regard to VSI and LI by neurologists (C.B. and K.J.). This examination was based on the clinical symptoms described in the medical records during their stay at the stroke unit, including speech therapist and neuropsychologist reports when applicable.

\section{Neurological deficits}

Neurological deficits were assessed by the SSS [19]. The analyses were made using the following two versions: the full SSS (score 0-58) and the SSS without the language subitem (score 0-48). In the latter version, the language item was excluded as this subitem was used as a separate variable to classify LI (described above). Lower scores indicate more deficits.

\section{Visual field deficit}

Visual field deficit was classified as either present or absent using the conventional confrontation technique. For 136 of the recruited patients, the classification was based on retrospective information from the medical records, examined by a neurologist (C.B.).

\section{Localization of acute stroke lesion}

All included participants had a brain computerized tomography examination and $215(91.5 \%)$ underwent brain magnetic resonance imaging. A neurologist (K.J.) reviewed the patients' computerized tomography/magnetic resonance imaging reports and classified acute stroke lesion as supratentorial (left, right or bilateral) or infratentorial. Previous infarcts were also documented. 


\section{Side of clinical symptoms}

The neurological clinical symptoms described in the medical records were classified by a neurologist (K.J.) as either left- or right-sided or as non-asymmetric.

\section{Stroke subtype}

All patients were classified by a neurologist (K.J.) according to the Oxfordshire Community Stroke Project classification criteria [20] as lacunar infarct, total anterior circulation infarct, partial anterior circulation infarct or posterior circulation infarct.

\section{Risk factors}

Risk factors for stroke were collected at inclusion. Hypertension, diabetes, hyperlipidemia and smoking are well-documented risk factors described in detail elsewhere [15]. These risk factors were rated as present or not present.

\section{Outcomes}

Outcomes 7 years after the index stroke were assessed with the modified Rankin scale (mRS) and Frenchay Activities Index (FAI). FAI is a self-rated questionnaire [21] assessing instrumental activities of daily living, based on the frequency of performance of activities with regard to domestic, leisure/work and outdoor activities. The total score of the scale based on 15 items was used in this study (range, 0-45). Additional information about these procedures is given in Data S1.

\section{Statistical analyses}

Spearman's rank-order correlation (Rho) was performed between baseline predictors and the total score for each of the two outcome variables. For descriptive purposes, the FAI total score was trichotomized. Predictors with a correlation coefficient $\geq 0.15$ were selected for a further multiple regression analysis. VSI and LI were selected for the multiple regressions regardless of their level in the bivariate correlation.

Categorical regression (CATREG) with optimal scaling was used as multiple regressions. The optimal scaling level, on which variables were analyzed in CATREG, was selected in accordance with their actual measurement level. For comparison, VSI and LI were coded as ordinal or nominal in two different analyses. No other optional function, except optimal scaling, was applied. CATREG with optimal scaling can transform non-linearly categorical data for predictors and a response variable. These transformed values (quantifications) were then used to find optimal numeric values to linearize the relation. An ordinary linear regression was then computed on these transformed scales. The choice of CATREG was verified by computing a traditional linear regression (without transformed scales) for the FAI total score, showing lower model fit and higher prediction error. All analyses were performed with SPSS Statistics, Version 21.0.

\section{Results}

Of the 375 recruited participants, 235 who had not experienced a recurrent stroke participated in the 7-year follow-up. Baseline data for these participants are shown in Table 1. Reasons for those lost to follow-up are given in Fig. 1. Baseline and follow-up

Table 1 Demographics and clinical data for the included participants

\begin{tabular}{lc}
\hline Baseline assessments & $(n=235)$ \\
\hline Age (years) & $56.0(12.0)$ \\
Females & $89(37.9)$ \\
VSI & \\
Lateralized visual inattention & $20(8.5)$ \\
Visual inattention & $27(11.5)$ \\
No visual inattention & $186(79.1)$ \\
LI & \\
Severe LI & $17(7.2)$ \\
Mild LI & $28(11.9)$ \\
No LI & $190(80.9)$ \\
SSS score full scale & $54.0(10.0)$ \\
SSS score without language item & $45.0(10.0)$ \\
Visual field deficit & $30(12.8)$ \\
CT/MRI results & \\
Localization of index stroke lesion & \\
$\quad$ Left hemisphere & $97(41.3)$ \\
$\quad$ Right hemisphere & $67(28.5)$ \\
$\quad$ Bilateral & $2(0.1)$ \\
$\quad$ Infratentorial & $48(20.4)$ \\
Old brain infarcts & $57(24.3)$ \\
Clinical symptoms & \\
Left-sided & $75(31.9)$ \\
Right-sided & $112(47.7)$ \\
Non-asymmetric & $48(20.4)$ \\
The Oxford Project Stroke Classification & \\
LACI & $78(33.2)$ \\
TACI & $23(9.8)$ \\
PACI & $62(26.4)$ \\
POCI & $69(29.4)$ \\
Not scored/other & $3(1.3)$ \\
Risk factors (yes) & \\
Hypertension & $122(51.9)$ \\
Diabetes & $40(17.0)$ \\
Hyperlipidemia & \\
Smoking & \\
\hline CT, conp. & \\
\hline
\end{tabular}

CT, computerized tomography; LACI, lacunar infarct; LI, language impairment; MRI, magnetic resonance imaging; PACI, partial anterior circulation infarct; POCI, posterior circulation infarct; SSS, Scandinavian Stroke Scale; TACI, total anterior circulation infarct; VSI, visuospatial inattention. Data are given as median (interquartile range) or $n(\%)$. 


\section{$\int$}

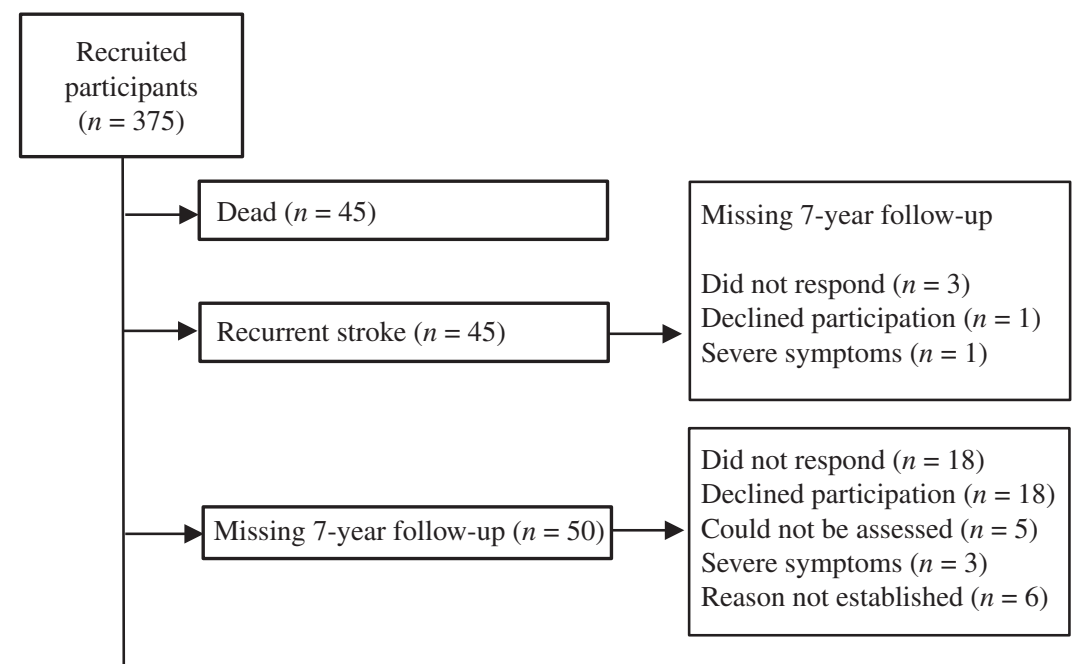

Included

participants

at 7-years

$(n=235)$

Figure 1 Flow chart of participants in the study.

data for included and excluded participants are given in Data S1 and Table S1.

Of the 235 participants, one did not return the FAI questionnaire at 7 years and 28 were not tested with the SCT for classifying VSI at baseline (12 due to severe impairments, eight recovered in the first few days, without further need for hospital care or outpatient rehabilitation, two were admitted to another department or hospital and the reason for a missing SCT could not be established for six patients). A retrospective classification of VSI could not be established for two patients and therefore 26 participants were retrospectively classified: nine as lateralized visual inattention, two as visual inattention and 15 as no visual inattention. The acute assessment of LI based on the SSS was not made for four patients and these cases were thus retrospectively classified, all as no LI.

\section{Baseline assessments and modified Rankin scale at 7 years}

Baseline variables according to the mRS score are shown in Table 2. No patients had a score of 5 at follow-up and therefore data for mRS scores of $0-4$ are given. Bivariate associations with an absolute correlation coefficient $\geq 0.15$ were observed for VSI, LI, for those who were retrospectively classified, SSS, total anterior circulation infarct and posterior circulation infarct. The multiple regression analysis (Table 3) identified VSI, the retrospectively classified and SSS as independent predictors for an increased mRS score at the 7-year follow-up. Similar results were obtained for both the analysis with the SSS without language $\left(R^{2}=0.435, P<0.001\right)$ and for the analysis with the full SSS $\left(R^{2}=0.414, \quad P<0.001\right)$. For additional analyses, see Table $\mathrm{S} 2$.

\section{Baseline assessments and Frenchay Activities Index at 7 years}

Baseline variables according to the FAI score are shown in Table 4. Bivariate associations between the predictors and FAI total score with an absolute correlation coefficient $\geq 0.15$ were observed for age, VSI, for those who were retrospectively classified, SSS, total anterior circulation infarct and diabetes. The multiple regression analysis identified VSI and SSS as independent significant predictors for the activity level in FAI (Table 5; $R^{2}=0.269, P<0.001$ ). The analysis with the full SSS also identified age and diabetes as significant predictors $\left(R^{2}=0.265\right.$, $P<0.001)$.

At the CATREG analyses, VSI and LI were coded as ordinal for mRS and nominal for FAI as these codings resulted in the best model fit for these variables.

\section{Discussion}

This study investigated whether a screening of VSI and LI at the early stage after stroke could provide prognostic information about long-term functional 
Table 2 Baseline assessments according to modified Rankin scale (mRS) scores at 7 years after index stroke

\begin{tabular}{|c|c|c|c|c|c|c|c|}
\hline \multirow[b]{2}{*}{ Baseline variable } & \multicolumn{5}{|c|}{ mRS score 7 years post-stroke } & \multicolumn{2}{|c|}{ Test statistics } \\
\hline & $\begin{array}{l}0 \\
47(20.0)\end{array}$ & $\begin{array}{l}1 \\
49(20.9)\end{array}$ & $\begin{array}{l}2 \\
91(38.7)\end{array}$ & $\begin{array}{l}3 \\
28(11.9)\end{array}$ & $\begin{array}{l}4 \\
20(8.5)\end{array}$ & Rho & $P$ \\
\hline Age (years) & $56.0(12.0)$ & $56.0(12.0)$ & $54.0(10.5)$ & $56.5(13.8)$ & $59.5(11.3)$ & 0.06 & 0.406 \\
\hline Females & $13(27.0)$ & $24(49.0)$ & $33(36.3)$ & $9(32.1)$ & $10(50.0)$ & 0.04 & 0.548 \\
\hline $\mathrm{VSI}^{\mathrm{a}, \mathrm{b}}$ & & & & & & -0.35 & $<0.001$ \\
\hline Lateralized visual inattention & $3(6.4)$ & 0 & $3(3.3)$ & $4(14.3)$ & $10(50.0)$ & & \\
\hline Visual inattention & $2(4.2)$ & $3(6.1)$ & $13(14.3)$ & $4(14.3)$ & $5(25.0)$ & & \\
\hline No visual inattention & $42(89.4)$ & $46(93.9)$ & $75(82.4)$ & $19(67.9)$ & $4(20.0)$ & & \\
\hline $\mathrm{LI}^{\mathrm{a}}$ & & & & & & -0.21 & 0.001 \\
\hline Severe LI & 0 & $1(2.0)$ & $6(6.6)$ & $7(25.0)$ & $3(15.0)$ & & \\
\hline Mild LI & $1(2.1)$ & $7(14.3)$ & $16(17.6)$ & $4(14.3)$ & 0 & & \\
\hline No LI & $46(97.9)$ & $41(83.7)$ & $69(75.8)$ & $17(60.7)$ & $17(85.0)$ & & \\
\hline Retrospectively classified for VSI or $\mathrm{LI}^{\mathrm{c}}$ & $4(8.5)$ & $6(12.2)$ & $5(5.5)$ & $6(21.4)$ & $9(45.0)$ & 0.22 & 0.001 \\
\hline \multicolumn{8}{|l|}{ Neurological deficits } \\
\hline SSS score full scale ${ }^{\mathrm{d}}$ & $57.0(4.0)$ & $54.0(9.5)$ & $54.0(7.8)$ & $45.0(28.0)$ & $28.0(25.0)$ & -0.45 & $<0.001$ \\
\hline SSS score without language item ${ }^{\mathrm{d}}$ & $47.0(3.5)$ & $46.0(9.5)$ & $45.0(6.0)$ & $37.0(28.0)$ & $18.0(21.0)$ & -0.42 & $<0.001$ \\
\hline Visual field deficit & $5(10.6)$ & $6(12.2)$ & $9(9.9)$ & $6(21.4)$ & $4(20.0)$ & 0.08 & 0.231 \\
\hline \multicolumn{8}{|l|}{ CT/MRI results } \\
\hline Localization of index stroke lesion $^{\mathrm{e}}$ & & & & & & 0.03 & 0.663 \\
\hline Left hemisphere & $17(36.2)$ & $24(49.0)$ & $37(40.7)$ & $13(46.4)$ & $6(30.0)$ & & \\
\hline Right hemisphere & $15(32.0)$ & $12(25.5)$ & $23(25.3)$ & $7(25.0)$ & $10(50.0)$ & & \\
\hline Bilateral & 0 & $1(2.0)$ & 0 & $1(3.6)$ & 0 & & \\
\hline Infratentorial & $9(19.1)$ & $9(18.4)$ & $23(25.3)$ & $6(21.4)$ & $1(5.0)$ & & \\
\hline Old brain infarcts & $13(27.7)$ & $9(18.4)$ & $22(24.2)$ & $7(25.0)$ & $6(30.0)$ & 0.02 & 0.778 \\
\hline Clinical symptoms ${ }^{\mathrm{e}}$ & & & & & & -0.02 & 0.798 \\
\hline Left-sided & $17(36.2)$ & $13(26.5)$ & $25(27.5)$ & $9(32.1)$ & $11(55.0)$ & & \\
\hline Right-sided & $19(40.4)$ & $25(51.1)$ & $45(49.4)$ & $15(53.6)$ & $8(40.0)$ & & \\
\hline Non-asymmetric & $11(23.4)$ & $11(22.4)$ & $21(23.1)$ & $4(14.3)$ & $1(5.0)$ & & \\
\hline \multicolumn{8}{|l|}{ The Oxford Project Stroke Classification } \\
\hline LACI & $21(44.7)$ & $17(34.7)$ & $24(26.4)$ & $9(32.1)$ & $7(35.0)$ & -0.09 & 0.152 \\
\hline TACI & 0 & 0 & $7(7.7)$ & $6(21.4)$ & $10(50.0)$ & 0.38 & $<0.001$ \\
\hline PACI & $9(19.1)$ & $13(26.5)$ & $30(32.9)$ & $8(28.6)$ & $2(10.0)$ & 0.03 & 0.685 \\
\hline POCI & $17(36.2)$ & $19(38.8)$ & $27(29.7)$ & $5(17.9)$ & $1(5.0)$ & -0.18 & 0.005 \\
\hline Not scored/other & 0 & 0 & $3(3.3)$ & 0 & 0 & - & - \\
\hline \multicolumn{8}{|l|}{ Risk factors (yes) } \\
\hline Hypertension & $23(48.9)$ & $28(57.1)$ & $46(50.5)$ & $13(46.4)$ & $12(60.0)$ & 0.01 & 0.932 \\
\hline Diabetes & 7 (14.9) & $4(8.2)$ & $16(17.6)$ & $8(28.6)$ & $5(25.0)$ & 0.13 & 0.047 \\
\hline Hyperlipidemia & $31(66.0)$ & $38(77.6)$ & $61(67.0)$ & $19(67.9)$ & $19(95.0)$ & 0.08 & 0.225 \\
\hline Smoking & $19(40.4)$ & $12(24.5)$ & $33(36.3)$ & $12(42.9)$ & $7(35.0)$ & 0.03 & 0.653 \\
\hline
\end{tabular}

The bivariate Rho was computed between the total outcome score and baseline scores or between the total outcome score and the two-level dichotomized baseline score. CT, computerized tomography; LACI, lacunar infarct; MRI, magnetic resonance imaging; PACI, partial anterior circula-

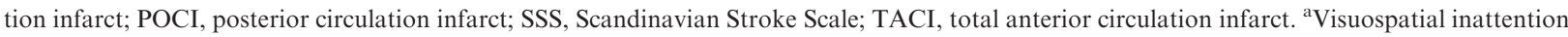
(VSI) was coded into three ordinal levels: lateralized visual inattention (1), visual inattention (2) and no visual inattention (3). Language impairment (LI) was coded into three ordinal levels: severe LI (1), mild LI (2) and no LI (3). ${ }^{\text {b }}$ Two patients who did not perform the Star Cancellation Test (SCT) could not be retrospectively classified for VSI. ${ }^{~}$ Patients who did not perform the SCT or SSS at baseline were retrospectively classified for the presence of VSI and LI. ${ }^{\mathrm{d}}$ Seven patients did not have a total score in SSS. CT/MRI showed signs of acute infarcts in $206(87.7 \%)$, whereas $16(6.8 \%)$ had a normal CT/MRI. ${ }^{e}$ Analyses of CT/MRI localization of acute stroke lesion and side of clinical symptoms were restricted to those with hemispheric lesions and asymmetric symptoms, respectively. Data are given as median (interquartile range) or $n(\%)$. Bold values indicate $P \leq 0.05$.

outcomes. Interestingly, we found that VSI, as assessed with a basic paper-and-pencil test, predicted outcomes 7 years after stroke, with respect to both disability and frequency of performance of activities of daily living, and also after controlling for LI and other neurological deficits. In contrast, screening of LI using the SSS did not provide independent prognostic information.
The negative impact of neglect on short-term functional outcome is well established in the literature [7]. With respect to long-term outcome, there are few studies on outcomes 1 year after stroke, all indicating worse outcomes for patients with neglect [6,14,22-27]. The current result adds information about the importance of neglect as a predictor of functional outcome several years after stroke, further emphasizing the 
Table 3 Predictors of modified Rankin scale at 7 years after index stroke

\begin{tabular}{|c|c|c|c|c|c|c|}
\hline & \multicolumn{2}{|c|}{$\begin{array}{l}\text { Standardized } \\
\text { coefficients }\end{array}$} & \multirow[b]{2}{*}{ Importance } & \multirow[b]{2}{*}{ d.f. } & \multirow[b]{2}{*}{$F$} & \multirow[b]{2}{*}{$P$} \\
\hline & $\beta$ & $\mathrm{SE}$ & & & & \\
\hline \multicolumn{7}{|c|}{ Analysis with SSS score without the language item } \\
\hline VSI & -0.306 & 0.112 & 0.370 & 2 & 7.455 & 0.001 \\
\hline LI & 0.101 & 0.071 & -0.027 & 2 & 2.000 & 0.138 \\
\hline Retrospectively classified for VSI or LI & 0.163 & 0.081 & 0.120 & 1 & 4.056 & 0.045 \\
\hline SSS score without language item & -0.308 & 0.069 & 0.351 & 2 & 19.947 & $<0.001$ \\
\hline Total anterior circulation infarct & 0.146 & 0.093 & 0.154 & 1 & 2.488 & 0.116 \\
\hline Posterior circulation infarct & 0.076 & 0.039 & 0.032 & 1 & 3.758 & 0.054 \\
\hline \multicolumn{7}{|l|}{ Analysis with full SSS } \\
\hline VSI & -0.285 & 0.114 & 0.357 & 2 & 6.296 & 0.002 \\
\hline Retrospectively classified for VSI or LI & 0.145 & 0.080 & 0.109 & 1 & 3.268 & 0.072 \\
\hline SSS score full scale & -0.300 & 0.072 & 0.360 & 2 & 17.246 & $<0.001$ \\
\hline Total anterior circulation infarct & 0.139 & 0.088 & 0.154 & 1 & 2.460 & 0.118 \\
\hline Posterior circulation infarct & 0.046 & 0.033 & 0.020 & 1 & 1.932 & 0.166 \\
\hline
\end{tabular}

Multiple regression with optimal scaling. Analysis with Scandinavian Stroke Scale (SSS) score without language item: $R^{2}=0.435, F=18.213$, degrees of freedom (d.f.) $=9, P<0.001$, adjusted $R^{2}=0.411$; analysis with full SSS: $R^{2}=0.414, F=21.725$, d.f. $=7, P<0.001$, adjusted $R^{2}=0.395$. Importance indicates the contribution of each predictor to the model (\%). This measure is similar to the product of the regression coefficient and zero-order correlation and can be used to reveal possible suppressor variables. Visuospatial inattention (VSI) was coded into three ordinal levels: lateralized visual inattention (1), visual inattention (2) and no visual inattention (3). Language impairment (LI) was coded into three ordinal levels: severe LI (1), mild LI (2) and no LI (3). Patients who did not perform the Star Cancellation Test or SSS at baseline were retrospectively classified for the presence of VSI and LI. Patients with missing values were excluded. SE, standard error. Bold values indicate $P \leq 0.05$.

importance of this symptom as a predictor of independence and activity. The SCT is quick to administer and feasible for most patients. Thus, our observations suggest that inclusion of a basic paper-and-pencil test of VSI at the post-acute stage can identify a group of patients at risk of unfavorable long-term outcomes in which targeted rehabilitative interventions may be indicated.

Although LI, as assessed with the SSS early poststroke, showed associations with long-term functional outcomes, it did not add any further prognostic information beyond the information provided by VSI and other neurological deficits. Data on LI as a predictor of long-term functional outcomes are limited $[9,14,28,29]$. Results from previous studies are equivocal, as two reported aphasia as a significant predictor of functional outcome $[9,28]$, whereas others were negative $[14,29]$. These studies used different assessments for aphasia early post-stroke, such as the SSS [9], Taylor Sarno test [14], Glasgow scale [28] or Boston Diagnostic Aphasia Examination criteria [29]. Our study, as well as others $[9,14,28,29]$, used general measures of basic and instrumental activities of daily living as functional outcomes. As early aphasic symptoms may be related to more language-specific aspects of functional outcome, it is important to recognize the possible limitation of these types of scales. Thus, at present, the value of aphasia as a predictor of long-term functional outcomes is uncertain. Further long-term studies, preferably with more comprehensive assessments of aphasia and more detailed outcome metrics, are needed.

One important methodological limitation of the present study is that we used rather basic measures for VSI and LI, which hampers a detailed description of different subtypes of neglect or aphasia. However, it is also an advantage as it limits the number of patients excluded due to inability to complete the tests. Another limitation is that some factors that may influence longterm outcome in young patients with stroke, such as epileptic seizures [30], were not included. Further limitations were that several neuropsychological functions, e.g. memory and executive functions, were not included in the current study and information about the symptoms of VSI and LI at discharge was not included. A particular strength of our study is that it consists of a consecutive series of patients with ischemic stroke admitted to a stroke unit and not a selective sample from a rehabilitation center. Another strength is that VSI and LI were investigated simultaneously early after stroke as predictors of long-term functional outcomes prospectively with a longitudinal design.

\section{Conclusion}

Taken together, in this study of young and middle-aged adults with ischemic stroke, we show that VSI, as assessed with a basic paper-and-pencil test at the postacute stage of stroke, is an independent predictor of 
Table 4 Baseline assessments according to Frenchay Activities Index (FAI) score at 7 years after index stroke

\begin{tabular}{|c|c|c|c|c|c|}
\hline \multirow{3}{*}{ Baseline variable } & \multicolumn{3}{|c|}{ FAI scores 7 years post-stroke ${ }^{a}$} & \multicolumn{2}{|c|}{ Test statistics } \\
\hline & $0-15$ & $16-30$ & $31-45$ & & \\
\hline & $39(16.7)$ & $121(51.7)$ & $74(31.6)$ & Rho & $P$ \\
\hline Age (years) & $58.0(11.0)$ & $57(11.0)$ & $52(13.0)$ & -0.20 & 0.002 \\
\hline Females & $13(33.3)$ & $44(36.4)$ & $31(41.9)$ & 0.09 & 0.178 \\
\hline $\mathrm{VSI}^{\mathrm{b}, \mathrm{c}}$ & & & & 0.30 & $<0.001$ \\
\hline Lateralized visual inattention & $10(25.6)$ & $9(7.4)$ & $1(1.4)$ & & \\
\hline Visual inattention & $7(17.9)$ & $15(12.4)$ & $5(6.8)$ & & \\
\hline No visual inattention & $20(51.3)$ & $97(80.2)$ & 68 (91.9) & & \\
\hline $\mathrm{LI}^{\mathrm{b}}$ & & & & 0.11 & 0.099 \\
\hline Severe LI & $6(15.4)$ & $10(8.3)$ & $1(1.4)$ & & \\
\hline Mild LI & $3(7.7)$ & 14 (11.6) & $11(14.9)$ & & \\
\hline No LI & $30(76.9)$ & $97(80.2)$ & $62(83.8)$ & & \\
\hline Retrospectively classified for VSI or $\mathrm{LI}^{\mathrm{d}}$ & $9(23.1)$ & $17(14.4)$ & $4(5.4)$ & -0.22 & 0.001 \\
\hline \multicolumn{6}{|l|}{ Neurological deficits } \\
\hline SSS score full scale $e^{e}$ & $39.0(27.0)$ & $54.0(10.0)$ & $55.0(6.3)$ & 0.33 & $<0.001$ \\
\hline SSS score without language item ${ }^{\mathrm{e}}$ & $29.0(27.0)$ & $46.0(9.0)$ & $46.0(5.5)$ & 0.32 & $<0.001$ \\
\hline Visual field deficit & $7(17.9)$ & $15(12.4)$ & $8(10.8)$ & -0.06 & 0.347 \\
\hline \multicolumn{6}{|l|}{ CT/MRI results } \\
\hline Localization of acute stroke lesion $^{\mathrm{f}}$ & & & & 0.05 & 0.569 \\
\hline Left hemisphere & $16(41.0)$ & $52(43.0)$ & $29(39.2)$ & & \\
\hline Right hemisphere & $13(33.3)$ & $27(22.3)$ & $26(35.1)$ & & \\
\hline Bilateral & $1(2.6)$ & 0 & $1(1.4)$ & & \\
\hline Infratentorial & $7(17.9)$ & $28(23.1)$ & $13(17.6)$ & & \\
\hline Old brain infarcts & $11(28.2)$ & $28(23.1)$ & $17(23.0)$ & -0.06 & 0.387 \\
\hline Clinical symptoms ${ }^{\mathrm{f}}$ & & & & -0.04 & 0.591 \\
\hline Left-sided & $16(41.0)$ & $33(27.3)$ & $25(33.8)$ & & \\
\hline Right-sided & $19(48.7)$ & $60(49.6)$ & $33(44.6)$ & & \\
\hline Non-asymmetric & $4(10.3)$ & $28(23.1)$ & $16(21.6)$ & & \\
\hline \multicolumn{6}{|l|}{ The Oxford Project Stroke Classification } \\
\hline LACI & $15(38.5)$ & $36(29.8)$ & $26(35.1)$ & 0.03 & 0.706 \\
\hline TACI & $13(33.3)$ & $8(6.6)$ & $2(2.7)$ & -0.28 & $<0.001$ \\
\hline PACI & $6(15.4)$ & $35(28.9)$ & $21(28.4)$ & 0.07 & 0.302 \\
\hline POCI & $5(12.8)$ & $40(33.1)$ & $24(32.4)$ & 0.10 & 0.151 \\
\hline Not scored/other & 0 & $2(1.6)$ & $1(1.4)$ & - & - \\
\hline \multicolumn{6}{|l|}{ Risk factors (yes) } \\
\hline Hypertension & $23(59.0)$ & $59(48.8)$ & $40(54.1)$ & -0.02 & 0.733 \\
\hline Diabetes & $11(28.2)$ & $26(21.5)$ & $3(4.1)$ & -0.25 & $<0.001$ \\
\hline Hyperlipidemia & $31(79.5)$ & $80(66.1)$ & $57(77.0)$ & 0.02 & 0.771 \\
\hline Smoking & $15(38.5)$ & $46(38.0)$ & $21(28.4)$ & -0.09 & 0.180 \\
\hline
\end{tabular}

Median (IQR) are given for ordinal data and proportions (\%) for nominal data. The bivariate Rho was computed between the total outcome score and baseline scores or between the total outcome score and the two-level dichotomized baseline score. CT, computerized tomography; LACI, lacunar infarct; MRI, magnetic resonance imaging; PACI, partial anterior circulation infarct; POCI, posterior circulation infarct; SSS, Scandinavian Stroke Scale; TACI, total anterior circulation infarct. ${ }^{a}$ Trichotomized FAI total score is only for descriptive purposes, whereas all analyses are based on FAI total score. ${ }^{b}$ Visuospatial inattention (VSI) was coded into three ordinal levels: lateralized visual inattention (1), visual inattention (2) and no visual inattention (3). Language impairment (LI) was coded into three ordinal levels: severe LI (1), mild LI (2) and no LI (3). ${ }^{\mathrm{c}}$ Two patients who did not perform the Star Cancellation Test (SCT) could not be retrospectively classified for VSI. ${ }^{\mathrm{d}}$ Patients

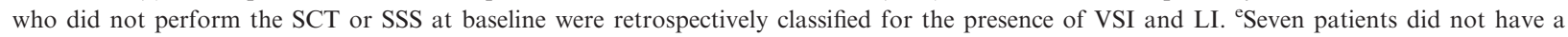
total score in SSS. Patients without assessment on FAI $(n=1)$. CT/MRI showed signs of acute infarcts in $205(87.6 \%)$, whereas $16(6.8 \%)$ had a normal CT/MRI. fAnalyses of CT/MRI localization of acute stroke lesion and side of clinical symptoms were restricted to those with hemispheric lesions and asymmetric symptoms, respectively. Bold values indicate $P \leq 0.05$.

functional outcomes 7 years later. These results emphasize the importance of the impact of VSI for resuming activity and independence after stroke. Thus, early recognition of VSI is of importance for rehabilitation planning and may enable targeted interventions.

\section{Acknowledgements}

The authors would like to express their gratitude to research nurse Ingrid Eriksson for her outstanding assistance with data collection. 
Table 5 Predictors of activity level (Frenchay Activities Index) at 7 years after index stroke

\begin{tabular}{|c|c|c|c|c|c|c|}
\hline & \multicolumn{2}{|c|}{$\begin{array}{l}\text { Standardized } \\
\text { coefficients }\end{array}$} & \multirow[b]{2}{*}{ Importance } & \multirow[b]{2}{*}{ d.f. } & \multirow[b]{2}{*}{$F$} & \multirow[b]{2}{*}{$P$} \\
\hline & $\beta$ & $\mathrm{SE}$ & & & & \\
\hline \multicolumn{7}{|l|}{ Analysis with SSS score without language item } \\
\hline Age & -0.128 & 0.068 & 0.070 & 1 & 3.534 & 0.061 \\
\hline VSI & 0.173 & 0.094 & 0.227 & 2 & 3.403 & 0.035 \\
\hline LI & 0.058 & 0.058 & 0.032 & 2 & 1.017 & 0.364 \\
\hline Retrospectively classified for VSI or LI & 0.052 & 0.059 & 0.038 & 1 & 0.785 & 0.377 \\
\hline SSS score without language item & 0.294 & 0.076 & 0.458 & 2 & 14.786 & $<0.001$ \\
\hline Total anterior circulation infarct & 0.067 & 0.071 & 0.079 & 1 & 0.901 & 0.344 \\
\hline Diabetes & 0.143 & 0.074 & 0.096 & 1 & 3.714 & 0.055 \\
\hline \multicolumn{7}{|l|}{ Analysis with full SSS } \\
\hline Age & -0.128 & 0.063 & 0.071 & 1 & 4.122 & 0.044 \\
\hline VSI & 0.169 & 0.090 & 0.227 & 2 & 3.515 & 0.031 \\
\hline Retrospectively classified for VSI or LI & 0.058 & 0.061 & 0.042 & 1 & 0.911 & 0.341 \\
\hline SSS score full scale & 0.299 & 0.075 & 0.476 & 2 & 15.993 & $<0.001$ \\
\hline Total anterior circulation infarct & 0.074 & 0.069 & 0.088 & 1 & 1.122 & 0.291 \\
\hline Diabetes & 0.142 & 0.069 & 0.097 & 1 & 4.262 & 0.040 \\
\hline
\end{tabular}

Multiple regression with optimal scaling. Analysis with Scandinavian Stroke Scale (SSS) score without language item: $R^{2}=0.269, F=7.750$, degrees of freedom (d.f.) $=10, P<0.001$, adjusted $R^{2}=0.234$; analysis with full SSS: $R^{2}=0.265, F=9.578$, d.f. $=8, P<0.001$, adjusted $R^{2}=0.237$. Importance indicates the contribution of each predictor to the model $(\%)$. This measure is similar to the product of the regression coefficient and zero-order correlation and can be used to reveal possible suppressor variables. Visuospatial inattention (VSI) was coded into three nominal levels: lateralized visual inattention, visual inattention and no visual inattention. Language impairment (LI) was coded into three nominal levels: severe LI, mild LI and no LI. Patients who did not perform the Star Cancellation Test or SSS at baseline were retrospectively classified for the presence of VSI and LI. Patients with missing values were excluded. SE, standard error. Bold values indicate $P \leq 0.05$.

This study received financial support from the Swedish Research Council (K2014-64X-14605-12-5) and grants from the Sahlgrenska University Hospital (ALFGBG-429981, ALFGBG-430841), Swedish Heart and Lung Foundation (20130315), Skaraborg Institute for Research and Development, Swedish Stroke Association, Rune and Ulla Amlövs Foundation for Neurological Research, John and Brit Wennerström Foundation for Neurological Research, Per-Olof Ahl Foundation for Neurological Research, Stroke Centre West, Gothenburg Foundation for Neurological Research, Hjalmar Svensson Research Foundation and Greta and Einar Askers Foundation.

\section{Disclosure of conflicts of interest}

The authors declare no financial or other conflicts of interest.

\section{Supporting Information}

Additional Supporting Information may be found in the online version of this article:

Data S1. Methods and results.

Table S1. Acute demographics, visuospatial inattention, language impairment, neurological deficits and 7-year follow-up assessments for included and not included participants.
Table S2. Bivariate and multiple analyses of odds ratios for modified Rankin scale score $>2$ at 7 years.

\section{References}

1. Appelros P, Karlsson GM, Seiger A, Nydevik I. Neglect and anosognosia after first-ever stroke: incidence and relationship to disability. $J$ Rehabil Med 2002; 34: 215-220.

2. Dickey L, Kagan A, Lindsay MP, et al. Incidence and profile of inpatient stroke-induced aphasia in Ontario, Canada. Arch Phys Med Rehabil 2010; 91: 196-202.

3. Engelter ST, Gostynski M, Papa S, et al. Epidemiology of aphasia attributable to first ischemic stroke: incidence, severity, fluency, etiology, and thrombolysis. Stroke 2006; 37: 1379-1384.

4. Pedersen PM, Jorgensen HS, Nakayama H, Raaschou $\mathrm{HO}$, Olsen TS. Aphasia in acute stroke: incidence, determinants, and recovery. Ann Neurol 1995; 38: 659-666.

5. Pedersen PM, Jorgensen HS, Nakayama H, Raaschou HO, Olsen TS. Hemineglect in acute stroke - incidence and prognostic implications. The Copenhagen Stroke Study. Am J Phys Med Rehabil 1997; 76: 122-127.

6. Appelros P, Karlsson GM, Seiger A, Nydevik I. Prognosis for patients with neglect and anosognosia with special reference to cognitive impairment. $J$ Rehabil Med 2003; 35: 254-258.

7. Jehkonen $\mathbf{M}$, Laihosalo $\mathbf{M}$, Kettunen JE. Impact of neglect on functional outcome after stroke: a review of methodological issues and recent research findings. Restor Neurol Neurosci 2006; 24: 209-215.

8. Nesi M, Lucente G, Nencini P, Fancellu L, Inzitari D. Aphasia predicts unfavorable outcome in mild ischemic 
stroke patients and prompts thrombolytic treatment. $J$ Stroke Cerebrovasc Dis 2014; 23: 204-208.

9. Tsouli S, Kyritsis AP, Tsagalis G, Virvidaki E, Vemmos KN. Significance of aphasia after first-ever acute stroke: impact on early and late outcomes. Neuroepidemiology 2009; 33: 96-102.

10. Wade DT, Hewer RL, David RM, Enderby PM. Aphasia after stroke: natural history and associated deficits. J Neurol Neurosurg Psychiatry 1986; 49: 11-16.

11. Gialanella B, Ferlucci C. Functional outcome after stroke in patients with aphasia and neglect: assessment by the motor and cognitive functional independence measure instrument. Cerebrovasc Dis 2010; 30: 440-447.

12. Paolucci S, Antonucci G, Gialloreti LE, et al. Predicting stroke inpatient rehabilitation outcome: the prominent role of neuropsychological disorders. Eur Neurol 1996; 36: 385-390.

13. Paolucci S, Antonucci G, Pratesi L, et al. Functional outcome in stroke inpatient rehabilitation: predicting no, low and high response patients. Cerebrovasc Dis 1998; 8: $228-234$

14. Giaquinto S, Buzzelli S, Di Francesco L, et al. On the prognosis of outcome after stroke. Acta Neurol Scand 1999; 100: 202-208.

15. Jood K, Ladenvall C, Rosengren A, Blomstrand C, Jern C. Family history in ischemic stroke before 70 years of age: the Sahlgrenska Academy Study on Ischemic Stroke. Stroke 2005; 36: 1383-1387.

16. Redfors P, Jood K, Holmegaard L, et al. Stroke subtype predicts outcome in young and middle-aged stroke sufferers. Acta Neurol Scand 2012; 126: 329-335.

17. Halligan PW, Cockburn J, Wilson BA. The behavioural assessment of visual neglect. Neuropsychol Rehabil 1991; 1: 5-32.

18. Samuelsson H, Hjelmquist E, Naver H, Blomstrand C. Different criteria in the assessment of visuospatial neglect. J Neurol Neurosurg Psychiatry 1995; 8: 114-115.

19. Boysen G. The Scandinavian Stroke Scale. Cerebrovasc Dis 1992; 2: 239-240.
20. Bamford J, Sandercock P, Dennis M, Burn J, Warlow C. Classification and natural history of clinically identifiable subtypes of cerebral infarction. Lancet 1991; 337: 1521-1526.

21. Holbrook M, Skilbeck CE. An activities index for use with stroke patients. Age Ageing 1983; 12: 166-170.

22. Jehkonen M, Ahonen JP, Dastidar $\mathrm{P}$, et al. Visual neglect as a predictor of functional outcome one year after stroke. Acta Neurol Scand 2000; 101: 195-201.

23. Jehkonen M, Ahonen JP, Dastidar P, et al. Predictors of discharge to home during the first year after right hemisphere stroke. Acta Neurol Scand 2001; 104: 136-141.

24. Katz N, Hartman-Maier A, Ring H, Soroker N. Functional disability and rehabilitation outcome in right hemisphere damaged patients with and without unilateral spatial neglect. Arch Phys Med Rehabil 1999; 80: 379-384.

25. Paolucci S, Grasso MG, Antonucci G, et al. Mobility status after inpatient stroke rehabilitation: 1 year-followup and prognostic factors. Arch Phys Med Rehabil 2001; 82: $2-8$.

26. Paolucci S, Grasso MG, Antonucci G, et al. One-year follow-up in stroke patients discharged from rehabilitation hospital. Cerebrovasc Dis 2000; 10: 25-32.

27. Young J, Bogle S, Forster A. Determinants of social outcome measured by the Frenchay Activities Index at one year after stroke onset. Cerebrovasc Dis 2001; 14: 114-120.

28. Taub NA, Wolfe CD, Richardson E, Burney PG. Predicting the disability of first-time stroke sufferers at 1 year. 12-month follow-up of a population-based cohort in southeast England. Stroke 1994; 25: 352-357.

29. Leśniak M, Bak T, Czepiel W, Seniów J, Czlonkowska A. Frequency and prognostic value of cognitive disorders in stroke patients. Dement Geriatr Cogn Disord 2008; 26: 356-363.

30. Arntz RM, Maaijwee NAM, Rutten-Jacobs LCA, et al. Epilepsy after TIA or stroke in young patients impairs long-term functional outcome. Neurology 2013; 81: 1907-1913. 